\title{
SEM and Raman Spectroscopy Applied to Biomass Analysis for Application in the Field of Biofuels and Food Industry
}

\author{
O. Samek ${ }^{1}$, A. Haronikova ${ }^{2}$, N. Vaskovicova ${ }^{1}$, K. Hrubanova ${ }^{1}$, J. Jezek $^{1}$, I. Marova ${ }^{2}$, V. Krzyzanek ${ }^{1}$, \\ P. Zemanek ${ }^{1}$ \\ ${ }^{1 .}$ Institute of Scientific Instruments of the ASCR, v.v.i., Brno, Czech Republic. \\ 2. Centre for Material Research, Brno University of Technology, Brno, Czech Republic.
}

A biomass of algal (Trachydiscus minutus, Botryococcus sudeticus, and Chlamydomonas sp.) and red yeast strains (Rhodotorula spp., Cystofilobasidium spp. and Sporobolomyces spp.) has been studied due to their potential applications in the field of biofuel generation and food industry [1-2]. In order to utilize biomass for efficient industrial production, the optimal cultivation parameters have to be determined which in turn lead to high production of desired substances such as oil and carotenoids in the selected cell line [2]. Main aim of our investigations was to study - using scanning electron microscopy (SEM) and Raman spectroscopy techniques - how different cultivation conditions influence production of oil and carotenoids. Raman spectroscopy can be used for the determination of the oil present in the biomass and also for the determination of carotenoids as the intensity ratios of specific, selected Raman bands [1]. SEM uses electron beams to gain information about morphology of cells (biomass structure) which is very important factor to study cells response on the applied stress.

In our experiments we have observed different morphology when employing SEM to study cells of Cystofilobasidium capitatum cultivating in the two media with different carbon to nitrogen $(\mathrm{C} / \mathrm{N})$ ratio (Figure 1). $\mathrm{C} / \mathrm{N}$ ratio of cultivation media has an influence to the production of yeast lipids or carotenoids [3]. First growth medium (medium 1) indicates positive results to carotenoid production and second medium (medium 2) with high $\mathrm{C} / \mathrm{N}$ ratio leads to an increased lipid production which can be indicated using Raman spectroscopy (Figure 2).

Samples for SEM imaging were prepared in the following way: (i) after thawing suspension of Cystofilobasidium capitatum (cultivated aerobically at $28^{\circ} \mathrm{C}$ on rotary incubator at $90 \mathrm{rpm}$ for 144 hours), the samples were further cultivated in the two different media for $24 \mathrm{~h}$ at temperature $37^{\circ} \mathrm{C}$, (ii) cells suspensions were processed by chemical fixation - 2 hours in $2.5 \%$ glutaraldehyde in PBS and $30 \mathrm{~min}$ in $1 \% \mathrm{OsO}_{4}$, dehydrated by acetone series and dried in HDMS on the glass slides. Both images of prepared samples were scanned without any metal coating at electron beam energy $1 \mathrm{keV}$ and beam current $3.1 \mathrm{pA}$ in SEM Magellan (FEI). Figure 1a shows ,clean/smooth“ surface of the cells (medium 1) contrary to the surface of the cells exposed to medium 2 where cells are most likely covered by lipid crystals. This could be explained by higher osmotic pressure introduced by medium 2 .

Here we combined information about morphology of the sample with Raman fingerprint by which lipid and carotenoids molecules can be identified. Thus, all matrix changes within the studied cells introduced by stress response mechanisms can be visualized (SEM) and chemically characterize (Raman). However, systematic studies are still required to investigate cell stress response mechanisms in more details. Such studies are currently under way in our laboratories, exploiting combination of SEM and Raman spectroscopy approaches. 
References:

[1] O. Samek et al, Sensors 10 (2010), p. 8635.

[2] I. Marova et al, J Environ Manag 95 (2012), p. S338.

[3] T. Braunwald et al, Appl Microbiol Biotechnol 97 (2013), p. 6581.

[4] This work received support from the Ministry of Health, Ministry of Education, Youth and Sports of the Czech Republic (LO1212) together with the European Commission and the Czech Science Foundation (ALISI No. CZ.1.05/2.1.00/01.0017) and the Grant Agency of the Czech Republic (GA14-20012S).
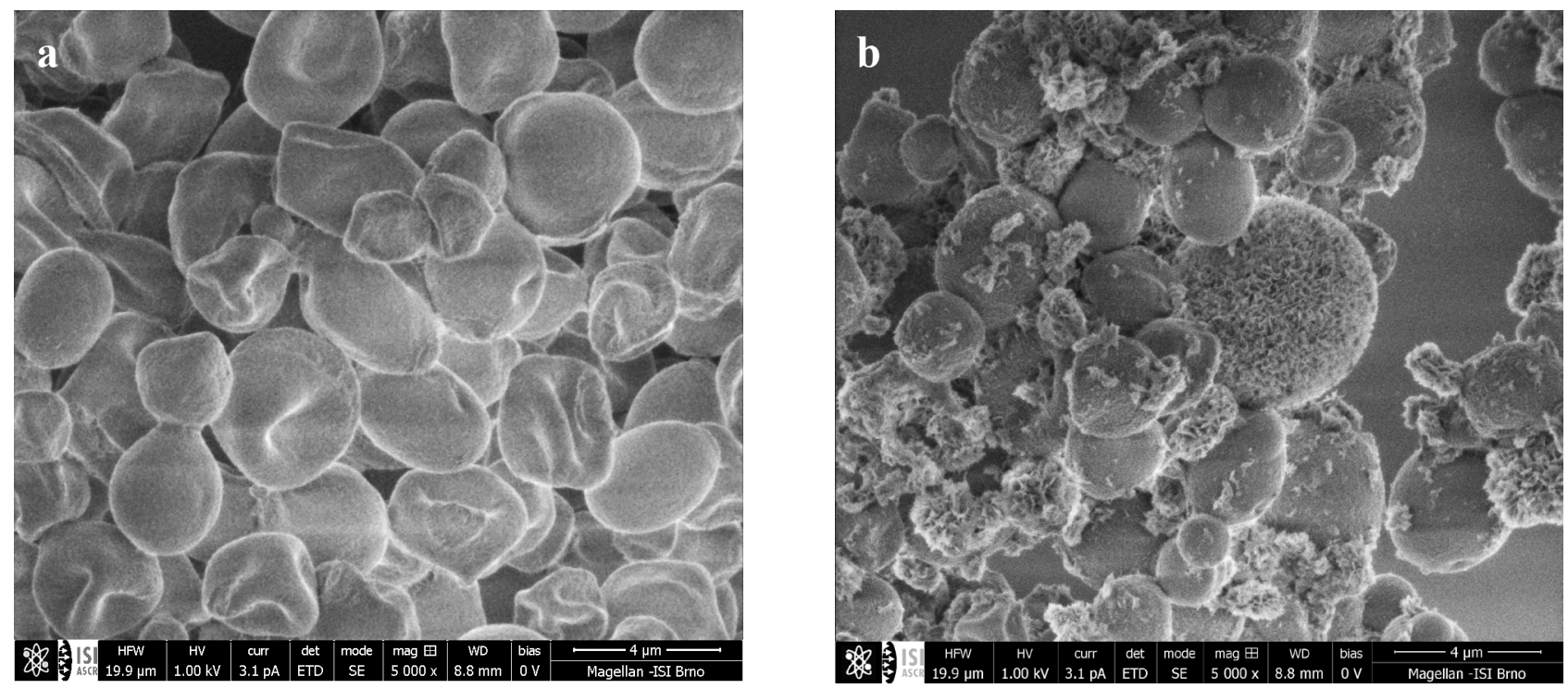

Figure 1. a) SEM image of Cystofilobasidium capitatum (medium 1); b) SEM image of Cystofilobasidium capitatum (medium 2).

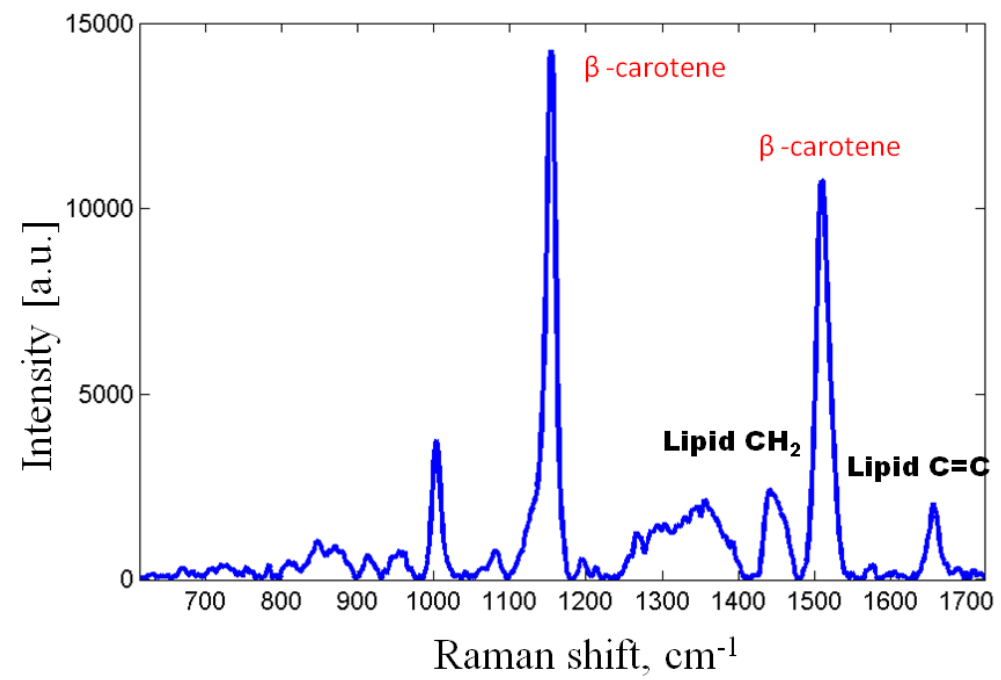

Figure 2. Raman spectra of Cystofilobasidium capitatum cultivated in medium 2 (increased lipid production in medium with high $\mathrm{C} / \mathrm{N}$ ration is clearly visible). 